\title{
Mechanical Characterization of III-V Nanowire Using Molecular Dynamics Simulation
}

\author{
Alex. W. Dawotola ${ }^{{ }_{1}}$, C. A. Yuan ${ }^{1}$, W. D. van Driel ${ }^{1,2}$, E. P. A. M. Bakkers ${ }^{3}$ and G. Q. Zhang ${ }^{1,2}$ \\ 1. Department of Precision and Microsystems Engineering, Delft University of Technology, The Netherlands \\ 2. NXP Semiconductors, Nijmegen, The Netherlands \\ 3. Philips Research, Eindhoven, The Netherlands \\ *a.w.dawotola@tudelft.nl
}

\begin{abstract}
Mechanical stiffness and density of III-V (GaAs) nanowire (NW) are studied by atomistic simulation in the $<111\rangle,\langle 110\rangle$ and $\langle 100\rangle$ directions. Series of molecular models are established and mechanical characteristics of the crystal orientations are considered. The simulation results indicate that the NW exhibits highest structural stiffness in the $\langle 111>$ direction. We also found that GaAs NW exhibits mechanical linearity under 2GPa stress. Moreover, a qualitative comparison of simulation and other calculated results is carried out, and a good agreement is established.
\end{abstract}

\section{Introduction}

Nanowires have many interesting properties that are not seen in bulk materials. This is because electrons in nanowires are quantum confined laterally and thus occupy energy levels that are different from the traditional continuum of energy levels or bands found in bulk materials. The mechanic response of nano materials are also found to be different from that of bulk materials [1]. There are different types of nanowires, including metallic (e.g., $\mathrm{Ni}, \mathrm{Au}$ ), semiconducting (e.g., InP, Si), and insulating types(e.g., $\mathrm{SiO}_{2}, \mathrm{TiO}_{2}$ ). III-V nanowires are semiconductor nanowires formed from a compound of group III and V elements. They have outstanding electronic and optical properties [2-3] and are considered ideal materials for photonic and electronic nanodevices, such as resonant tunneling diodes, single electron transistors, photoemitters, and photodetectors [4].

In order to guarantee a long-term reliability of the device, the mechanical response of the III- $V$ nanowire should be well understood. However, many mechanical properties of III-V NWs have not been thoroughly elucidated. Important issues in current research include understanding the influence of growth technologies and device processing on the final properties of the nanowire. Experimental techniques such as scanning tunneling microscopy (STM), atomic force microscopy (AFM), transmission electron microscopy (TEM) and nanoindentation have so far been employed in the mechanical characterization of III-V NWs [5-7]. However, in the absence of experimental data, atomistic simulation is expected to give a very good insight into their properties [8]. A simulation approach could aid the understanding of experiments as well as stimulate new experiments through its predictive power [9-10]. Moreover, one way to compliment the understanding gained from experimental methods is through the use of computer simulations, such as molecular dynamics (MD) and Monte Carlo (MC) simulations.

The Young's modulus, E of a rod in tension and the flexural rigidity, EI of a beam undergoing bending are one of the most fundamental mechanical properties of an engineering structure [11]. Through these properties, the stiffness of the material can be estimated. Molecular Dynamics simulation has proved to be effective technique for mechanical characterization of bulk systems on the atomic level [12]. In this work, MD simulation is applied to predict the stiffness (Young's modulus) and density of III-V (GaAs) NW in the $\langle 111\rangle,\langle 110\rangle$ and $\langle 100\rangle$ directions.

Although, GaAs nanowire is anisotropic, it is often desirable to define its Young's moduli as common in isotropic materials. A molecular model which describes the crystal orientation of the nanowire is established and a feasible loading and boundary condition is applied. The Young's modulus of the structure is extracted by analysing the force-displacement response produced in the dynamics of the structure. The density of the structure is also calculated.

\section{MD Simulation using ESFF Forcefield}

In brief, molecular dynamics simulation method is based on Newton's equation of motion, given by:

$$
\vec{f}_{i}=m_{i} \vec{a}_{i}
$$

Where $\vec{f}_{i}$ is the total force exerted on particle $i, m_{i}$ and $a_{i}$ are the mass and acceleration of particle $i$ respectively.

Force, $\vec{f}_{i}$ can also be expressed as the gradient of potential energy,

$$
\overrightarrow{f_{i}}=-\nabla_{i} V
$$

These two equations are combined to obtain a comprehensive expression:

$$
-\frac{d V}{d \vec{r}_{i}}(i=1, \ldots, N)=m_{i} \frac{d^{2} \vec{r}_{i}}{d t^{2}}
$$

where $V$ is the potential energy of the system. 
Based on this expression, Newton's equation of motion can then relate the derivative of potential energy to changes in position as a function of time. Equation (3) is integrated by discretizing time with an interval, $\Delta t$ and applying a finite-difference integrator that depends on statistical ensemble. In this paper, the microcanonical ensemble (NVE) ensemble, which conserves the number of atoms $(\mathrm{N})$, the system volume and the total energy, is used. Moreover, the velocity-Verlet algorithm is implemented.

For MD simulation, two kinds of information are required. The first is the chemical composition and spatial configuration of the atoms. The second is the forcefield, which defines the mechanical interactions between atoms. The choice of a forcefield determines to a great extent the accuracy of MD simulation. Therefore, an appropriate forcefield should be selected, to predict within a reasonable accuracy the potentials of the atoms. Among the available forcefields in atomistic simulation, the extensible systematic force field (ESFF) is chosen because it is well parameterized for applications to groups III and $\mathrm{V}$ elements and their compounds. The ESFF, proposed by Shenghua et al [13], is a rule-based forcefield covering a wide range of atoms. The ESFF is validated for structural characterization of some metallic type elements [14].

In the ESFF, atoms are parameterized using ab initio calculations and fitting of crystal structures. The parameters of the atoms are classified based on the types of interactions involved, which are; bond, angle, torsion, out-of-plane, electrostatic, and van der Waals interaction. The total energy $E$ is expressed as a sum of bond energy $E_{b d}$, angle energy $E_{a}$, torsion energy $E_{t}$, out-ofplane energy $E_{o p}$, van der Waals energy $E_{v d w}$ and electrostatic energy $E_{e s}$.

$$
E=E_{b d}+E_{a}+E_{t}+E_{o p}+E_{v d w}+E_{s}
$$

The bond types are characterized by the bond orders, $\pi$ lone-pair interactions, and symmetry positions. The bond energy, $\mathrm{kcal} / \mathrm{mol}$ is expressed in terms of a Morse function:

$$
E_{b d}=D_{i}\left[1-e^{-\alpha_{i}\left(r-r_{i}\right)}\right]^{2}
$$

where $\mathrm{r}$ is the distance between the atoms, $r_{i}$ is the equilibrium bond distance, $D_{i}$ is the "equilibrium" dissociation energy of the molecule (measured from the potential minimum), and $\alpha_{i}$ controls the 'width' of the potential and is equal to the square root of half the force constant divided by $D_{i}$,

$$
\alpha_{i}=\sqrt{k / 2 D_{i}}
$$

The angle energy, unit $\mathrm{kcal} / \mathrm{mol}$ varies for different types of angles and is classified according to coordination number, symmetry, $\pi$-bonding situation, and ring information, if applicable. For a linear angle $\theta$, the angle energy in terms of the force constant, $k$ of the atoms is given by [13]:

$$
E_{a}=k \cos ^{2} \theta
$$

Torsion types are determined by the central bond order and the torsion energy is calculated only if the central bond involves nonmetal atoms with coordination numbers less than 4. Similarly, the out-of-plane energy is included only when the centre atom is an $\mathrm{sp} 2$ hybridized nonmetal atom with more than two bonds or is an atom with $\mathrm{D}_{3 \mathrm{~h}}$ or $\mathrm{D}_{4 \mathrm{~h}}$ bonding symmetry having less than 5 bonds.

The van der Wals energy expression in ESFF is of Lennard-Jones 6-9 form [13,15-16]:

$$
E_{v d w}=\sum_{i}^{j} \sum_{j \neq i} \varepsilon_{i j}\left(2 \frac{r^{9}{ }_{v i j}}{r^{9}{ }_{i j}}-3 \frac{r^{6}{ }_{v i j}}{r^{6}{ }_{i j}}\right)
$$

where $r_{v i j}$ and $\mathcal{E}_{i j}$ are the van de Wals parameters, and are calculated from the atomic van der Waals radii and well depths of atoms $i$ and $j$.

The electrostatic energy is calculated by Coulomb's law:

$$
F=k_{c} \frac{\left|q_{i}\right| \cdot\left|q_{j}\right|}{r_{i j}^{2}}
$$

where: $k_{c}=1 / 4 \pi \varepsilon_{0}$ is the electrostatic constant and $\varepsilon_{0}$ is the electric constant (permittivity of free space), $r_{i j}$ is the distance between atom $i$ and atom $j$ and $q_{i}$ and $q_{j}$ are atomic partial charges, on atoms $i$ and $j$.

\section{Approach}

Fig. 1 below gives an overview of modeling and simulation procedures followed in this paper. 


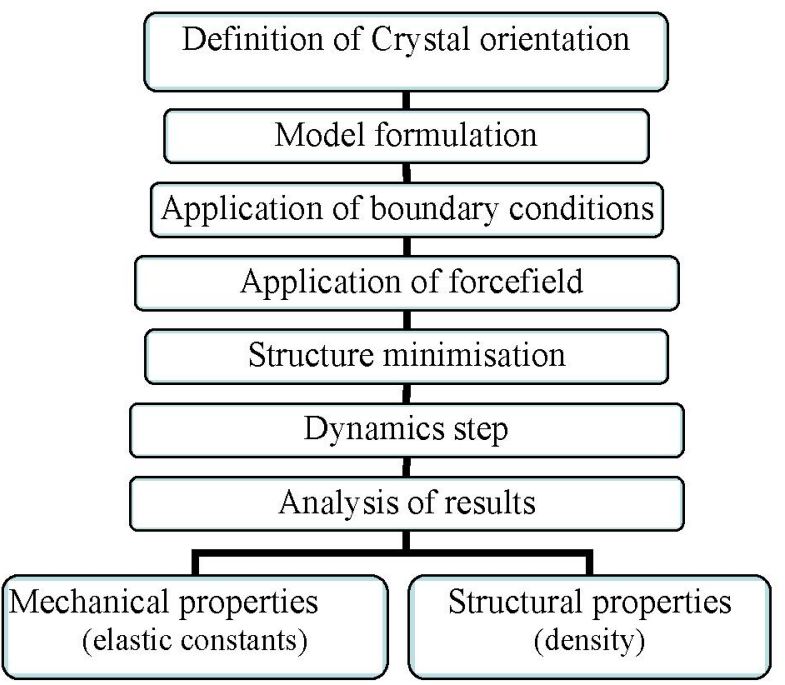

FIG. 1. Simulation steps: a step by step approach to the modelling and simulation process.

With sufficient description of the configuration and potential functions of the molecules, simulation results can quantitatively and qualitatively match experimental results and also interpret the mechanics of a molecular model under external loadings [17-18]. Moreover, the MD simulation results can assist the material scientist to develop a robust material with higher mechanical strength and longer reliability cycles by adjusting the material composition as desired.

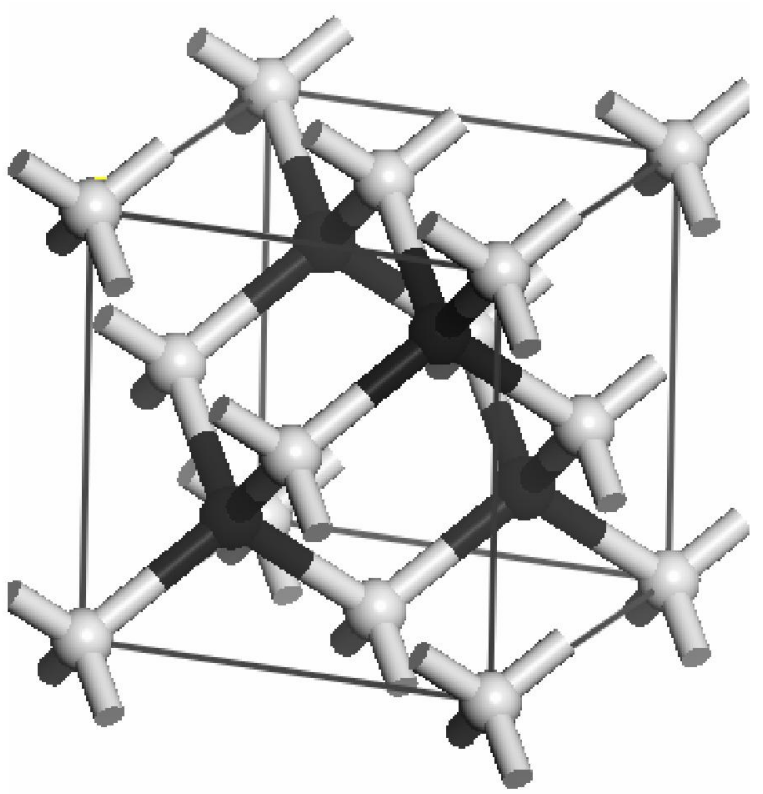

FIG. 2. Crystalline structure of GaAs. Arsenic and gallium atoms are coloured black and gray, respectively.

Modelling and simulation are carried out on the commercial software MS modeling 4.0 by Accelrys [19]. The NW model is developed from a sigle crystal of GaAs (which is zinc blende at room temperature, Fig. 2). The structure is minimized under a minimization step of 15,000 cycles and then stretched in the $x$-direction. The dynamics time is set at $200 \mathrm{ps}$. The simulation is carried out at room temperature, which is kept constant throughout the simulation with the aid of a Berendsen thermostat.

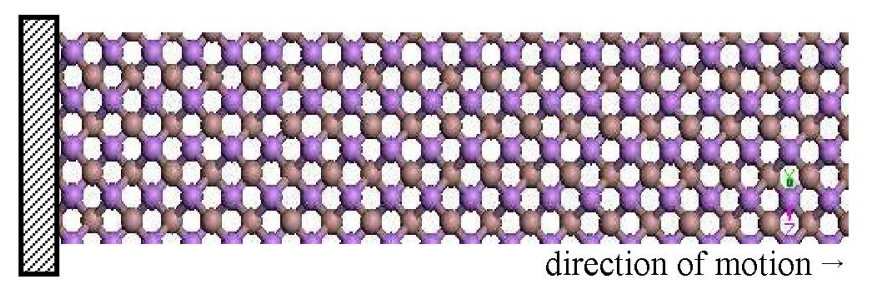

FIG. 3. The GaAs nanowire model.

In the model, two atom layers are fixed in all directions while the opposite end is in displacement.

The Force-displacement $(\mathrm{F}-\mathrm{dx})$ response curves are obtained after the dynamics step of the MD simulation, (Figs. 4, 5 and 6). The Young's modulus is calculated from the elasticity theory:

$$
E=\frac{F L}{A d x}
$$

where, $A$ and $L$ are the geometrical properties of the structure, (area and length, respectively). Furthermore, density of the NW is calculated from the ratio of atomic mass to molecular volume.

\section{Results}

GaAs NW models, which represent three different crystal orientations, all of area $1.5 \mathrm{~nm}^{2}$ and length $10 \mathrm{~nm}$, are established. The III-V nanowires tend to grow in the $<111>$ direction [21], making it the most important growth direction. However, we investigated the $\langle 110\rangle$ and $\langle 100\rangle$ directions to further compare the stiffness of the NW along different directions. The predicted Young's moduli for the NW along directions: $\langle 111\rangle,\langle 110\rangle$ and $<100>$ (using 1,000atoms) are $198.87 \mathrm{GPa}, 153.54 \mathrm{GPa}$ and $147.7 \mathrm{GPa}$ respectively. The result shows that GaAs NW is indeed anisotropic and has highest stiffness in the $<111>$ direction, and least in the $<100\rangle$ direction, due to the condensed packing of the atoms. This inference is further confirmed from the stress-strain plots, which show that a stress of $2 \mathrm{GPa}$ applied on the NW strains the material by $1 \%, 1.25 \%$ and $1.5 \%$ in the $\langle 111\rangle,\langle 110\rangle$ and $\langle 100\rangle$ directions respectively, confirming the ability of the NW to withstand more stress in the $\langle 111\rangle$ direction. The value of the density obtained, $4.96 \mathrm{~g} / \mathrm{cm}^{3}$ is also comparable, though lesser to the bulk value $(5.3 \mathrm{~g} / \mathrm{cm} 3)$. Our results show a qualitative agreement, of less than $45 \%$ deviation with calculated Young's moduli for GaAs by Brantley, W.A [20], 141.2GPa for $<111>$, $121.3 \mathrm{GPa}$ for $\langle 110\rangle$ and $85.3 \mathrm{GPa}$ for $\langle 100\rangle$, and we observed a similar trend in values. 


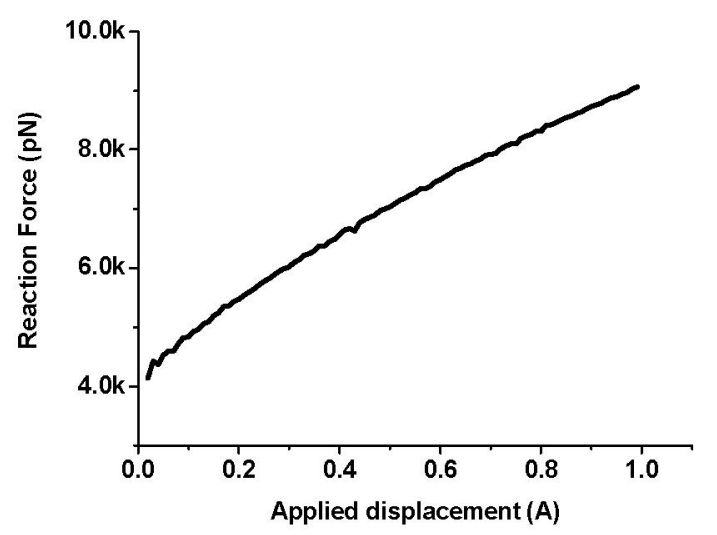

(a)

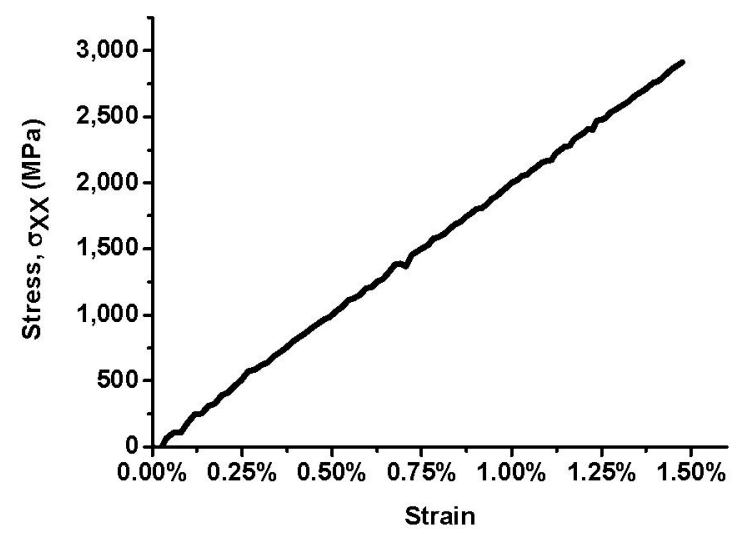

(b)

FIG. 4. Mechanical response of GaAs $<111>$ nanowire (a) Force-displacement response curve. (b) is the stress-strain curve.

\section{Conclusions}

In this paper, the mechanical and structural properties of III-V nanowire are computed using MD simulation. The simulation predicts the Young's moduli for GaAs nanowire in the three directions, $\langle 111\rangle,\langle 110\rangle$ and $<100\rangle$. The difference in our results and other numerical values [20] could be due to the size of the material simulated, area $1.5 \mathrm{~nm}^{2}$ and length $10 \mathrm{~nm}$, and system error from the forcefield. In our future research, we will investigate the influence of point defects, and size variation on the mechanical properties of III-V nanowires. We also hope to carry out a sensitivity analysis of these effects on the final properties of our NW model.

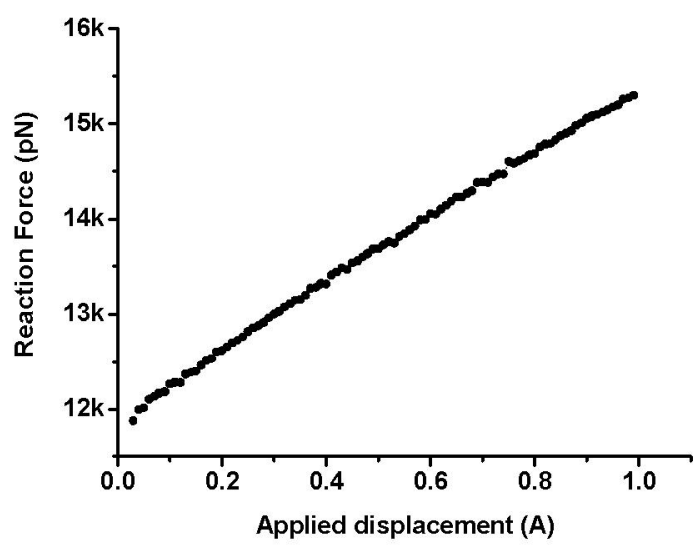

(a)

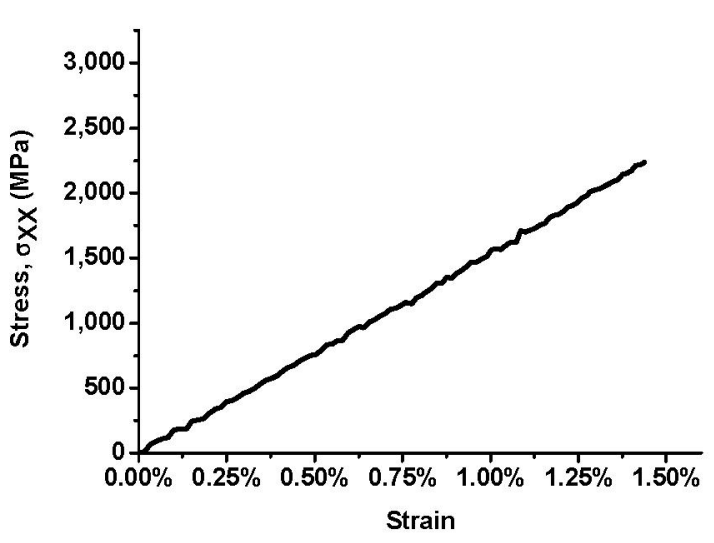

(b)

FIG. 5. Mechanical response of GaAs $<110>$ nanowire (a) Force-displacement response curve. (b) is the stress-strain curve.

\section{Acknowledgments}

A.W.D and C.AY are grateful to Dr J. Wescott and Dr C. Menke (from Accelrys) for their helpful discussions on III-V MD simulations. A.W.D would also like to appreciate the Netherlands Government for a graduate fellowship. 


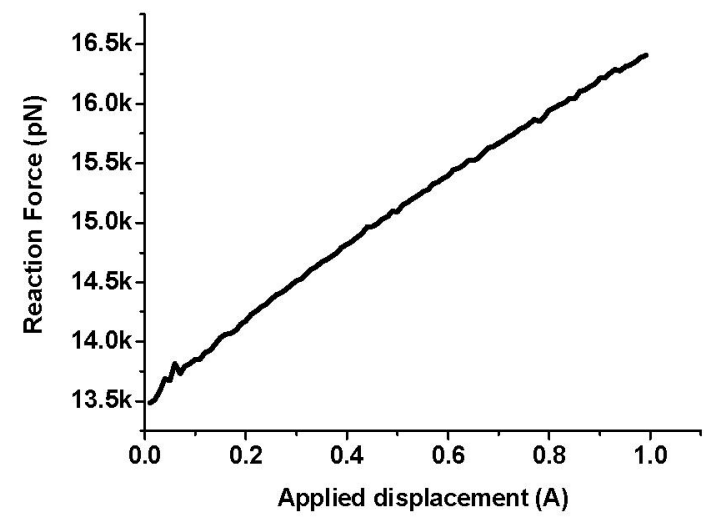

(a)

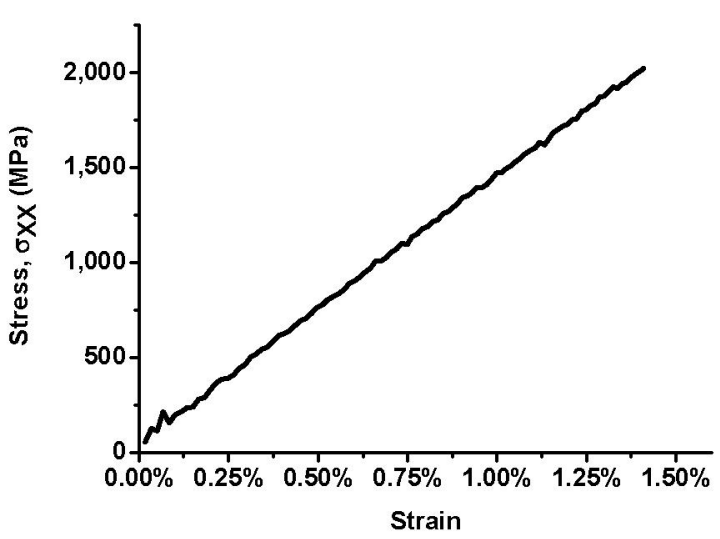

(b)

FIG. 6. Mechanical response of $\mathrm{GaAs}<100>$ nanowire. (a) Force-displacement response curve. (b) is the stressstrain curve.

\section{References}

1. B. Lee and R. E.Rudd, "First-principles study of the Young's modulus of $\mathrm{Si}<001>$ nanowires, eprint arXiv:cond-at/0611073 (2006)

2. X. Duan et al, "Indium phosphide nanowires as building blocks for nanoscale electronic and optoelectronic devices", Nature 409, 66-69 (2001)

3. M.Law et al "semionductor nanowires and nanotubes", Rev. Mater. Res.34, 83 (2004)

4. P. Paiano et al "Size and shape control of GaAs nanowires grown by metalorganic vapor phase epitaxy using tertiarybutylarsine", J. Appl. Phys. 100, 094305 (2006)

5. M. Tabib-Azar et al., "Mechanical properties of selfwelded silicon nanobridges", Appl Phy. Ltts., 87 (11), (2005).

6. Z.L. Wang et al., "Measuring the Young's modulus of solid nanowires by in situ TEM", J. of Electron microscopy 51, (2002)
7. Li XD et al, "Direct nanomechanical machining of gold nanowires using a nanoindenter and an atomic force microscope, J. micromech. and microengineering. 15 (3): 551-556 (2005).

8. S. Kodiyalam et al "grain boundaries in gallium arsenide nanocrystals under pressure: a parallel molecular dynamics study" Phys Rev Lett, 86, 55 (2000)

9. E. Z. da Silva et al, "Gold nanowires and the effect of impurities, Nano. Res Lett 1:91-98. (2006)

10. M. P. Allen, "Introduction to molecular dynamics simulation" in Computational Soft Matter: From Synthetic Polymers to Proteins, NIC Series, Vol. 23, pp. 1-28, (2004.)

11. R. Miller and V. Shenoy, "size dependent elastic properties of nano-sized structural elements", Nanotech., vol. 11, No.3, pp. 139-147 (2000)

12. M. Cheng and Y. Lu Structural stability of carbon nanotubes using molecular dynamics and finitedifference time-domain methods, IEEE, Vol 42. Iss 4, pp. 891-894 (2006).

13. S. Shi et al "An extensible and systematic force field, ESFF, for molecular modeling of organic, inorganic, and organometallic systems" J Comp. Chem. 24 No 9 1059-1076 (2003)

14. N. Jager and U. Schilde, "Molecular Mechanics calculations on Chelates of Titanium(IV), Vanadium(IV/V), $\quad$ Copper(II), Nickel(II), Molybdenum(IV/V), Rhenium(IV/V) and Tin(IV) with Di- and Tridentate Ligands Using the New Extensible Systematic Force Field (ESFF) - An Empirical Study", Str. Chemistry. Vol.9. No.2 (1998).

15. D. White "A computationally efficient alternative to the Buckingham potential for molecular mechanics calculations", J. Comp.-aided Mol. Design, Vol. 11, No. 5 (1997)

16. H. Tempel and M.Wolf "Third virial coefficient of ${ }^{4} \mathrm{He}$ for Lennard-Jones (6-9) potential in the temperature range from $18^{\circ} \mathrm{K}$ to $295^{\circ} \mathrm{K}$ ", Phys Ltts., A, Vol. 24, Iss. 3, pp. 187-188. (1967)

17. K. N. Chiang et al, "Mechanical Characteristic of ssDNA/dsDNA molecule under external loading", Appl. Phy. Lett., Vol. 88, pp. 023902, (2006).

18. C. Yuan et al, "Investigation of the sequencedependent dsDNA mechanical behavior using clustered atomistic-continuum Method," in proc. NSTI Nano conf., California, U.S.A.(2005).

19. Accelrys Inc., Material Studio ${ }^{\text {TM }}$ DISCOVER, San Diego: Accelrys Inc., (2005)

20. W. A. Brantley, "Calculated elastic constants for stress problems associated with semiconductor devices", J. Apply. Phys. Vol. 44, No.1 (1973)

21.M. Verheijen et al, "Growth kinetics of heterostructured GaP-GaAs nanowires", J. Am. Chem. Soc. 128 (4), 1353-1359 (2006). 